\title{
The New Approach to the Diagnostics and Treatment of Endogenous Intoxication in Patients with Burn Injury
}

\author{
Stepan Zaporozhan ${ }^{1}$, Vasyl Savchyn², Lesia Ostapiuk ${ }^{3}$, Anatolii Voloshinovskii ${ }^{4}$, \\ Nataliia Tuziuk ${ }^{1,2}$, Taras Malyi ${ }^{4}$ \\ ${ }^{1}$ Horbachevskyi Ternopil National Medical University, Ternopil, Ukraine \\ ${ }^{2}$ Lviv's Communal Clinical Hospital No. 8, Lviv, Ukraine \\ ${ }^{3}$ Lviv Regional Public Health Center, Lviv, Ukraine \\ ${ }^{4}$ Ivan Franko National University of Lviv, Lviv, Ukraine \\ Email: *lesya_ost@ukr.net
}

How to cite this paper: Zaporozhan, S., Savchyn, V., Ostapiuk, L., Voloshinovskii, A., Tuziuk, N. and Malyi, T. (2020) The New Approach to the Diagnostics and Treatment of Endogenous Intoxication in Patients with Burn Injury. International Journal of Clinical Medicine, 11, 375-388. https://doi.org/10.4236/ijcm.2020.116033

Received: May 12, 2020

Accepted: June 6, 2020

Published: June 9, 2020

Copyright $\odot 2020$ by author(s) and Scientific Research Publishing Inc. This work is licensed under the Creative Commons Attribution International License (CC BY 4.0).

http://creativecommons.org/licenses/by/4.0/

\begin{abstract}
Background: The main causes of mortality in patients with burn injury are the development of systemic inflammatory process, multiple organ failure and septic complications. The aim of the research: Improvement of diagnostic and therapeutic approaches to the detection and elimination of endogenous intoxication in patients with burn injury. Materials and methods: The main study group consisted of 19 patients and the comparison group-of 10 patients with burn injury. 92 blood serum (BS) samples of the patients of the main group were tested, using the method of fluorescence spectroscopy (MFS). The advanced therapeutic tactics were proposed for the patients of both groups. The control group consisted of 40 healthy individuals (donors). BS of these patients was also tested using MFS. Results: Patients with burn injury have endogenous intoxication in their blood. The effective concentration of albumin is reduced in patients with burn injury due to the blockage of albumin binding centers by bacterial metabolism products. Fluorescence spectra (FS) of BS in patients with burn trauma and donors were obtained and investigated. Based on MFS results, an improved treatment regimen using infusion of albumin solution was proposed. Conclusions: An improved technique for the management of patients with burn injury is based on the use of MFS for the diagnostic evaluation of endogenous intoxication in them. The idea of pathological changes in albumin molecules in patients with burn injury is pathogenetically substantiated by the successful use of infusion of albumin solution in these patients on the basis of the MFS.
\end{abstract}




\section{Keywords}

Burn Injury, Method of Fluorescence Spectroscopy, Endogenous

Intoxication, Albumin

\section{Introduction}

According to WHO, the problem of burn injury is currently one of the most global in the field of health care. It is estimated, that up to 180,000 burn deaths occur annually in the world, most of which occur in low- and middle-income countries, including Africa, South and East Asia. The mortality rate of infant patients with burn injuries is over 7 times higher in low-income countries than in highly developed countries. Burn injury is one of the main causes of morbidity, leading to prolonged hospitalization, distortion and disability, and is often accompanied by stigma and rejection. This is especially true for patients with head and neck burns [1] [2]. Therefore, the problem of burn injury causes a number of serious economic, social and psychological problems and requires the development of effective methods of medical and social rehabilitation of patients.

Despite the introduction into medical practice the modern achievements of resuscitation, the latest methods of intensive care, the incidence of multiple organ failure, septic complications and mortality among patients with common burns remain quite high [3]. The main factors of mortality are the development of systemic inflammatory process, multiple organ failure and infectious complications [3]. The fraction of deaths from burn disease in the stage of burn shock is $23 \%-39 \%$, in the stage of acute burn toxemia-35\% - $42 \%$, and in the stage of septicotoxemia-25\% - 30\%.

At the same time, a number of important theoretical and practical problems remain studied insufficiently. Also, the controversial are the issues about the radicality of early surgical interventions in patients with head and face burns, the extent and time of their performance, the means of plastic wound closure and the correction of general homeostasis disorders. The role of local and general disorders, characterizing the severity of traumatic injury, is also described insufficiently.

Therefore, the problem of recovery and survival of patients with burn injury is currently very relevant, important and not enough resolved. Therefore, many scientific researchers are devoted to this problem. However, insufficient attention was focused on assessing the prognosis for the development of purulent-septic complications in patients with burn injury and for their early diagnosis capabilities, especially monitoring and treatment.

In order to develop the pathogenetic approach of diagnosis, monitoring, management of the treatment process and prognosis, it is promising to use physical research methods, in particular MFS. Its high diagnostic value, accuracy and sensitivity makes it possible to build the concept of diagnostics, monitoring and elimination of endogenous intoxication in patients with burn trauma, which 
will significantly improve the survival and recovery of patients with burn injury.

\section{Literature Review}

The immediate cause of lethality in patients with severe burns is purulent-septic complications, which appear from damage of many organs and systems during the development of severe burn disease and septicotoxemia. Fatal complications are caused not by immediate burn injury, but by the body's reactive response to a thermal stimulus. It is based on the implementation of the local and then a generalized inflammatory response, mediated by the number of proinflammatory cytokines. Delay in the provision of intensive care in the stage of burn shock, delay in surgical repair of the skin, failure to identify and to neutralize microbial agents with the use of antimicrobial agents without local action also play a significant role in this process.

Burn necrotic scab is a source of infection and intoxication, so it should be removed as soon as possible, before the development of severe endogenous intoxication [3]. Therefore, the concept of early surgical necrectomies of burn wounds with their primary plasticity is rational, as well as the development of means for the prevention and treatment of wound infection, the restoration of anatomical structures and non-surgical correction in the postoperative period [1] [2]. However, the mechanism of the formation of endogenous intoxication in patients with burn injury was not fully established yet. There is no thorough understanding of the pathogenetic changes, that occur in burn injury, which does not allow to form an effective treatment strategy.

It should be noted, that because of presence of endogenous intoxication, conformational changes of the albumin BS molecules occur due to their interaction with toxins [4] [5]. Within the MFS, the excitation of BS was performed at a wavelength of $280 \mathrm{~nm}$, which corresponds to the excitation region of human serum albumin.

Pathologically altered albumin causes changes in the fluorescence spectrum of the BS, which we detect within our research. This method allows to record these changes 24 - 48 hours before the onset of pronounced clinical manifestations [6]. It was successfully used for the early diagnosis of sepsis (patent of Ukraine $\mathrm{N}^{\circ} 76953$ ) [7] and postpartum purulent-inflammatory diseases (patent of Ukraine $\mathrm{N}^{\circ} 33472$ ) [8]. In scientific publications [9] [10], we tested the use of MFS for the diagnosis of endogenous intoxication in patients with burn injury, taking into account similar mechanisms of its formation in sepsis and burn injury, despite various etiological factors.

The aim of the research is to improve the diagnostic and therapeutic approach to the detection and elimination of endogenous intoxication in patients with burn injury.

\section{Data and Methodology}

The clinical base of the research was the burn department of Lviv's Communal 
Clinical Hospital No. 8, and the experimental base-the laboratory of luminescence of the Department of Experimental Physics, Ivan Franko National University of Lviv. The term of the research was 2015-2020 years. Three groups of patients were formed. The main study group consisted of 19 patients with burn injury, for whom 92 BS samples were examined within the MFS. Inclusion criteria-patients with flame burns and burns with boiling of first- and second-degree (type A and B), with a total area of $18 \%$ to $45 \%$ of the body surface, including the head and neck. 11 patients $(60 \%)$ of the main group were in serious condition and $8(40 \%)$ were patients of moderate severity. Object of study-BS of patients with burn injury. The standard treatment algorithm for these patients was supplemented with infusion of albumin solution. The comparison group consisted of 10 patients, whose BS were not tested using MFS. 6 patients $(60 \%)$ of the comparison group were in serious condition and $4(40 \%)$ were patients of moderate severity. But therapeutic tactics with albumin solution was also used for these patients. The control group of the research consisted of 40 healthy individuals (donors) without chronic diseases, for whom BS samples were tested within the MFS.

Research methods: clinical, general blood test, biochemical, microbiological, morphological (structure of the areas of lesions, scars), immunological (immunohistochemistry of the skin), cytology of the wound, encephalography, ultrasound, doppler ultrasound, examination of microenvironment, computed tomography, densitometry, rheovasography, MFS.

\subsection{Data Source}

In the framework of this research, we investigated and analyzed the main factors, including clinical data, classification of burns, depending on the etiological factor, area and depth of lesion, localization, laboratory examination data (general blood test, general analysis of urine, biochemical blood test, bacterioscopic examination), ultrasonographic examination, MFS.

\subsection{Research Results}

During our research, we analyzed clinical data, results of laboratory, instrumental examinations and spectral-fluorescence characteristics of patients of the main group, group of comparison and spectral-fluorescence parameters of healthy controls. The main indicators that were used for the analysis of FS of BS were the fluorescence intensity $\left(\mathrm{I}_{\mathrm{F}}\right)$ and the position of the maximum fluorescence band $\left(\lambda_{\max }\right)$. All patients underwent surgical treatment of the affected burn surfaces with subsequent wound closure by lyophilized xenografts in the hospital. The wounds were epithelized partly under dry skin, partly under dry necrosis and applicators. The residual wounds were epithelialized under dry applicators. Patients also received anti-inflammatory treatment, antibiotic therapy, infusion therapy, including using albumin solution and desensitizing therapy. After successful completion of treatment, all patients were discharged from the hospital in 
satisfactory condition under the supervision of the surgeon at the place of residence. Now let's focus on some of the results obtained for patients of the main group in serious condition. To compare the spectral-fluorescence characteristics of BS of patients with burn injury, we shall also present in the relevant figures the results of the spectral-fluorescence characteristics of a patient with sepsis [6], who recovered after successful treatment.

The results of studies in the dynamics FS of BS and data for the spectral-fluorescence characteristics of the BS of patient with burn injury are depicted on Figure 1 and Table 1. He was admitted to the hospital on the $27^{\text {th }}$ of June, 2015 with the area of the burn surface $38 \%$. Staphyloccus aureus $10^{5}$ and Pseudomonas aeruginosa $10^{6}$ were verified in this patient on the basis of the microbiological study. He was immediately prescribed appropriate treatment, including antibiotic therapy and infusion therapy with a volume of $2-3$ liters daily and $20 \%$ albumin solution ( $100 \mathrm{ml} 8$ times in different days). Due to the infusion therapy, the intensity of FS of BS compared with the fluorescence intensity of albumin $\left(I_{F}=1.00\right)$ did not decrease significantly for several days $\left(I_{F}=0.88\right)$, which correlates with the results of in vitro studies [11]. At the same time, no significant shift of the FS of BS into the longwave region was recorded, despite the verification of several pathogens. Obviously, the intake of sufficient albumin allowed to improve significantly the work of detoxification systems of the body, which had a positive effect on the spectral-fluorescence parameters. Measurements of FS of BS after 10 days after admission to the hospital on the $13^{\text {th }}$ of July, 2015 (Figure 1, curve 1.3), testified to a critical moment, when there was a significant decrease in $\mathrm{I}_{\mathrm{F}}$ to 0.35 r.u. and the shift of the FS into the longwave region by $9 \mathrm{~nm}$.

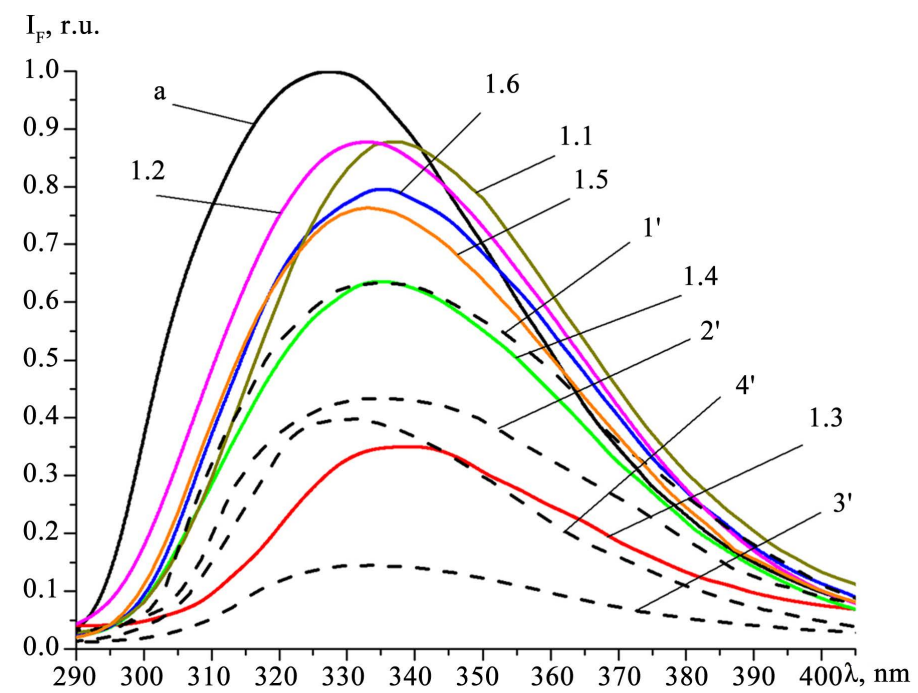

Figure 1. FS of BS of the patient 1 with burn injury, who was hospitalized at Lviv's Communal Clinical Hospital No. 8 in 2015 in dynamics during treatment $(1.1-3.07$, $1.2-8.07,1.3-13.07,1.4-17.07,1.5-20.07,1.6-24.07)$ and a patient with sepsis, who was treated in 2002 in Ambulance hospital (1'-03.06, 2'-05.06, 3'-06.06, 4'-07.06) and $20 \%$ albumin solution (a), $\lambda_{\text {ex }}=280 \mathrm{~nm}$. 
Table 1. Spectral-fluorescence parameters (fluorescence intensity $\left(\mathrm{I}_{\mathrm{F}}\right)$ and the position of the maximum fluorescence band $\left.\left(\lambda_{\max }\right)\right)$ of blood serum of patient 1 .

\begin{tabular}{cccccccccccc}
\hline $\mathrm{N}$ & $\mathrm{a}$ & 1.1 & 1.2 & 1.3 & 1.4 & 1.5 & 1.6 & 1 ' & 2' & $3^{\prime}$ & $4^{\prime}$ \\
\hline Date & 3.07 & 3.07 & 8.07 & 13.07 & 17.07 & 20.07 & 24.07 & 03.06 & 05.06. & 06.06 & 07.06 \\
$\lambda_{\max } \mathrm{nm}$ & 327 & 336.1 & 332.2 & 341.1 & 335.1 & 333.1 & 335.1 & 335.2 & 335.2 & 334.1 & 331.6 \\
I, r.u. & 1 & 0.88 & 0.88 & 0.35 & 0.64 & 0.76 & 0.80 & 0.63 & 0.43 & 0.14 & 0.40 \\
\hline
\end{tabular}

These parameters correlate well with the corresponding results of the study of FS of patient with sepsis, depicted in this figure by dashed lines (curves 1' - 4'), which is most likely related to the discontinuation of the albumin solution [12]. The resumption of infusion of albumin solution with subsequent complex therapy led to a gradual improvement of the spectral-fluorescence parameters of BS (approximated to the parameters of albumin fluorescence) at the subsequent blood samples during $17^{\text {th }}-24^{\text {th }}$ of July, 2015. They correlated well with the clinical parameters and laboratory test results of the patient. Therefore, he was discharged from the hospital in satisfactory condition on the $24^{\text {th }}$ of July 2015.

In Figure 2, there are depicted the results of studies in the dynamics of fluorescence spectra, and in Table 2-data for the spectral-fluorescence characteristics of the BS of another patient with burn injury, who was admitted to the hospital on the $27^{\text {th }}$ of June, 2015.

The area of the burn surface of this patient was $31 \%$. In this case, the patient had no bacteremia. This led to a different character of the changes in the spectral-fluorescence characteristics of BS. No significant decrease in the fluorescence intensity of the BS was observed and no significant long-wave shift was recorded. After two infusions of $20 \%$ solution of albumin $\left(28^{\text {th }}\right.$ of June $-400 \mathrm{ml}$ and $29^{\text {th }}$ of June $-200 \mathrm{ml}$ ) on the $3^{\text {rd }}$ of July the primary measurement of his spectral-fluorescence characteristics was done. Thus, it was obtained $\mathrm{I}_{\mathrm{F}}=0.66$ r.u. and shift in the longwave region to $339 \mathrm{~nm}$ (Table 2).

During the continuation of treatment (massive infusion therapy and $100 \mathrm{ml}$ of $20 \%$ albumin solution on the $13^{\text {th }}$ of July), slight changes in the spectral-fluorescence characteristics of BS were recorded. After the cancellation of treatment, the patient's condition gradually stabilized (on the $24^{\text {th }}$ of July $\mathrm{I}_{\mathrm{F}}=0.87$ r.u.) (Table 2) and he was discharged from the hospital on the $24^{\text {th }}$ of July, 2015 in satisfactory condition.

In a slightly different scenario, there was a change in the spectral-fluorescence characteristics of the BS of the next patient with burn injury, with a burn surface area $40 \%$. The results of the study of FS of this patient are depicted in Figure 3, and in Table 3-data for his spectral-fluorescence characteristics. On the basis of microbiological research, there were verified the presence of two pathogens (Staphyloccus aureus $5 \times 10^{6}$, St. haemolyticus $5 \times 10^{6}$ ). This patient received infusion, antibacterial therapy, as well as infusion of $20 \%$ solution of albumin (total volume $1000 \mathrm{ml}$ ). For this patient on the $8^{\text {th }}$ of July, 2015 (curve 3.1), a marked decrease in the fluorescence intensity was recorded to 0.53 r.u. 


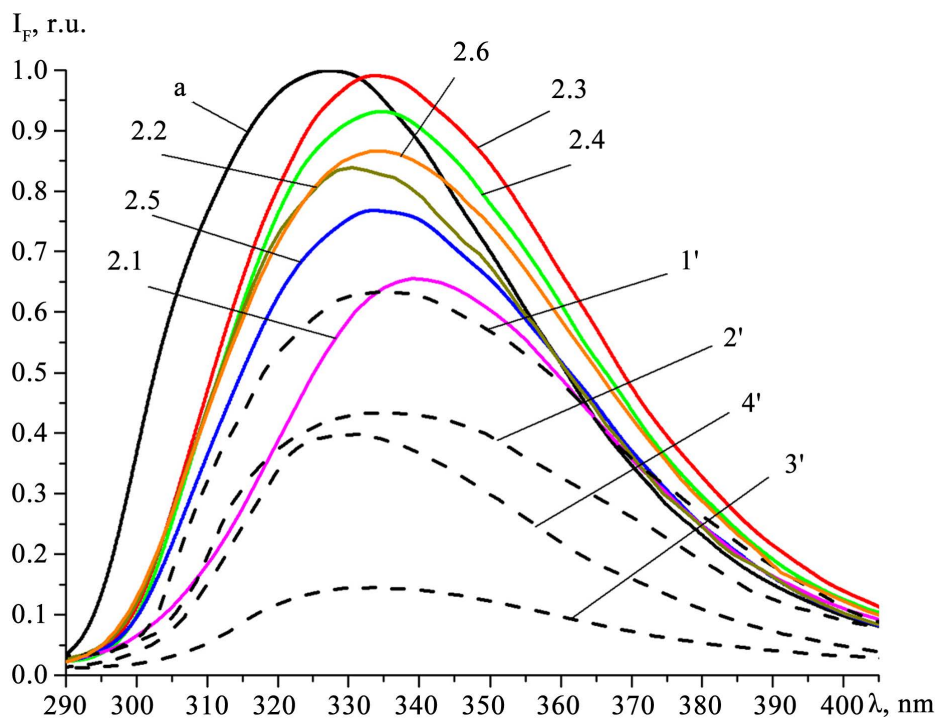

Figure 2. FS of BS of the patient 2 with burn injury, who was hospitalized at Lviv's Communal Clinical Hospital No. 8 in 2015 in dynamics during treatment $(2.1-3.07$, 2.2-8.07, 2.3-13.07, 2.4-17.07, 2.5-20.07, 2.6-24.07) and a patient with sepsis, who was treated in 2002 in Ambulance hospital (1'-03.06, 2'-05.06, 3'-06.06, 4'-07.06) and $20 \%$ albumin solution (a), $\lambda_{\mathrm{ex}}=280 \mathrm{~nm}$.

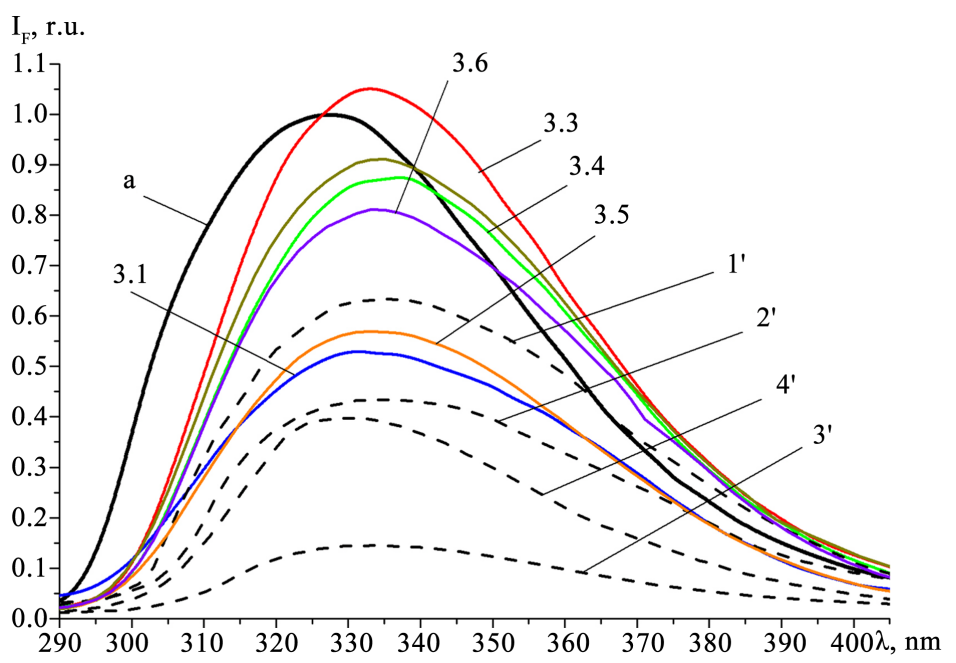

Figure 3. FS of BS of the patient 3 with burn injury, who was hospitalized at Lviv's Communal Clinical Hospital No 8 in 2015 in dynamics during treatment $(3.1-8.07$, 3.3-15.07, 3.4-17.07, 3.5-24.07, 3.6-28.07), and a patient with sepsis, who was treated in 2002 in Ambulance hospital (1'-03.06, 2'-05.06, 3'-06.06, 4'-07.06) and 20\% albumin solution (a), $\lambda_{\mathrm{ex}}=280 \mathrm{~nm}$.

Table 2. Spectral-fluorescence parameters (fluorescence intensity $\left(I_{F}\right)$ and the position of the maximum fluorescence band $\left(\lambda_{\max }\right)$ ) of blood serum of patient 2 .

\begin{tabular}{cccccccccccc}
\hline $\mathrm{N}$ & $\mathrm{a}$ & 2.1 & 2.2 & 2.3 & 2.4 & 2.5 & 2.6 & $1^{\prime}$ & $2^{\prime}$ & $3^{\prime}$ & $4^{\prime}$ \\
\hline Date & 3.07 & 3.07 & 8.07 & 13.07 & 17.07 & 20.07 & 24.07 & 03.06 & 05.06 & 06.06 & 07.06 \\
$\lambda_{\max } \mathrm{nm}$ & 327 & 339 & 330.5 & 333.1 & 335.1 & 333.1 & 335.1 & 335.2 & 335.2 & 334.1 & 331.6 \\
I, r.u. & 1 & 0.66 & 0.84 & 0.99 & 0.93 & 0.77 & 0.87 & 0.63 & 0.43 & 0.14 & 0.40 \\
\hline
\end{tabular}


Table 3. Spectral-fluorescence parameters (fluorescence intensity $\left(\mathrm{I}_{\mathrm{F}}\right)$ and the position of the maximum fluorescence band $\left.\left(\lambda_{\max }\right)\right)$ of blood serum of patient 3 .

\begin{tabular}{ccccccccccc}
\hline $\mathrm{N}$ & $\mathrm{a}$ & 3.1 & 3.3 & 3.4 & 3.5 & 3.6 & 1 & $2^{\prime}$ & $3^{\prime}$ & $4^{\prime}$ \\
\hline Date & 3.07 & 8.07 & 15.07 & 17.07 & 24.07 & 28.07 & 03.06 & 05.06 & 06.06 & 07.06 \\
$\lambda_{\max } \mathrm{nm}$ & 327 & 332 & 333.1 & 337.1 & 333.1 & 333.1 & 335.2 & 335.2 & 334.1 & 331.6 \\
I, r.u. & 1 & 0.53 & 1.05 & 0.88 & 0.57 & 0.81 & 0.63 & 0.43 & 0.14 & 0.40 \\
\hline
\end{tabular}

Further in dynamics on the $15^{\text {th }}$ of July, 2015 there was an increase in the fluorescence band of the patient's BS $\left(I_{F}=1.05\right.$ r.u., Table 3$)$, which cannot be interpreted as absolute hypoproteinemia, which typically causes a decrease in the fluorescence concentration quenching inherent in transient fluorescence [11]. Thereafter, there was a gradual decrease in the fluorescence intensity of the BS to $\mathrm{I}_{\mathrm{F}}=0.57$ r.u. $\left(24^{\text {th }}\right.$ of July, 2015). In the future, the patient's condition has stabilized $\left(\mathrm{I}_{\mathrm{F}}=0.81\right.$ r.u. $)$ and he was discharged from the hospital.

Particularly noteworthy are those depicted in Figure 4 the results of the study in the dynamics of FS of the BS of the patient with combined body trauma, concussion, multiple laceration wounds of the frontal parietal region, chest slaughter, lung slaughter, left hemothorax, abdominal wall slaughter, traumatic detachment of the left lower third of left thigh, shock of the third-grade and sepsis.

The results of the spectral-fluorescence characteristics of her BS are presented in Table 4. It was important for us to study in dynamics the changes of FS of the BS of this patient and to compare them with the corresponding results of patients with burn injury. Based on the microbiological study, the presence of three pathogens was verified for this patient (Ps. aeryginosa $1 \times 10^{5}$, Staphyloccus aureus $1 \times 10^{4}$ and Klebsiella pneumoniae $\left.1 \times 10^{4}\right)$.

The effective treatment was immediately prescribed for this patient, including antibiotic therapy and infusion therapy with a volume of 2 - 3 liters every day, including a $20 \%$ solution of albumin ( $100 \mathrm{ml}$ and $260 \mathrm{ml}$ for 2 days).

The effective treatment was immediately prescribed to this patient. It included antibiotic and infusion therapy with a volume of 2 - 3 liters every day, including $20 \%$ albumin solution ( $100 \mathrm{ml}$ and $260 \mathrm{ml}$ for 2 days).

Due to the large amount of infusion therapy, the fluorescence intensity of the BS was not reduced (curve 4.1), which correlates with the results of the in vitro study and the results of the BS study of the severe burn patients, described above. Subsequently, the fluorescence intensity decreased to $I_{F}=0.76$ r.u. (curve 4.3). On the background of further treatment, there was a significant improvement in the patient's condition $\left(I_{F}=0.98\right.$ r.u., curve 4.5$)$ and she was discharged from the hospital. It should be noted, that according to the results of our studies, the condition of this patient was much easier compared to the conditions of previous patients.

The results of FS of BS of two more patients with burn injury are depicted on Figure 5 and Figure 6. The corresponding results for the spectral-fluorescence characteristics for their BS are presented in Table 5 and Table 6 . They were 


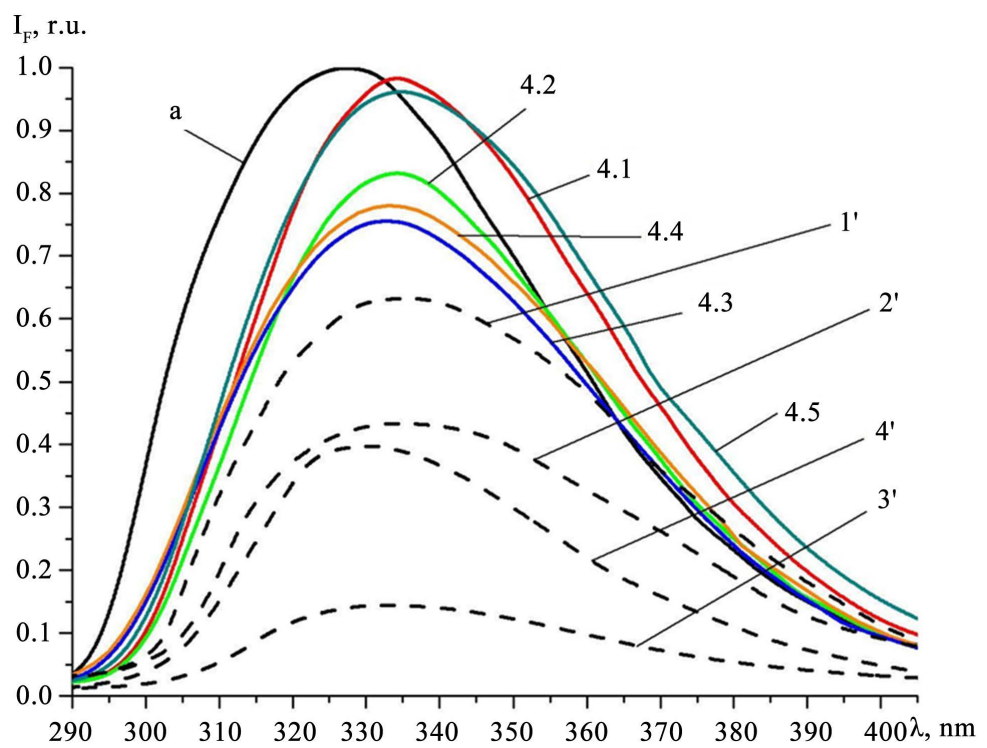

Figure 4. FS of BS of the patient 4 with combined body trauma, who was hospitalized at Lviv's Communal Clinical Hospital No 8 in 2015 in dynamics during treatment (7.1-17.07, 7.2-20.07, 7.3-24.07, 7.4-28.07, 7.5-31.07), and a patient with sepsis, who was treated in 2002 in Ambulance hospital (1'-03.06, 2'-05.06, 3'-06.06, $4-07.06$ ) and $20 \%$ albumin solution (a), $\lambda_{\mathrm{ex}}=280 \mathrm{~nm}$.

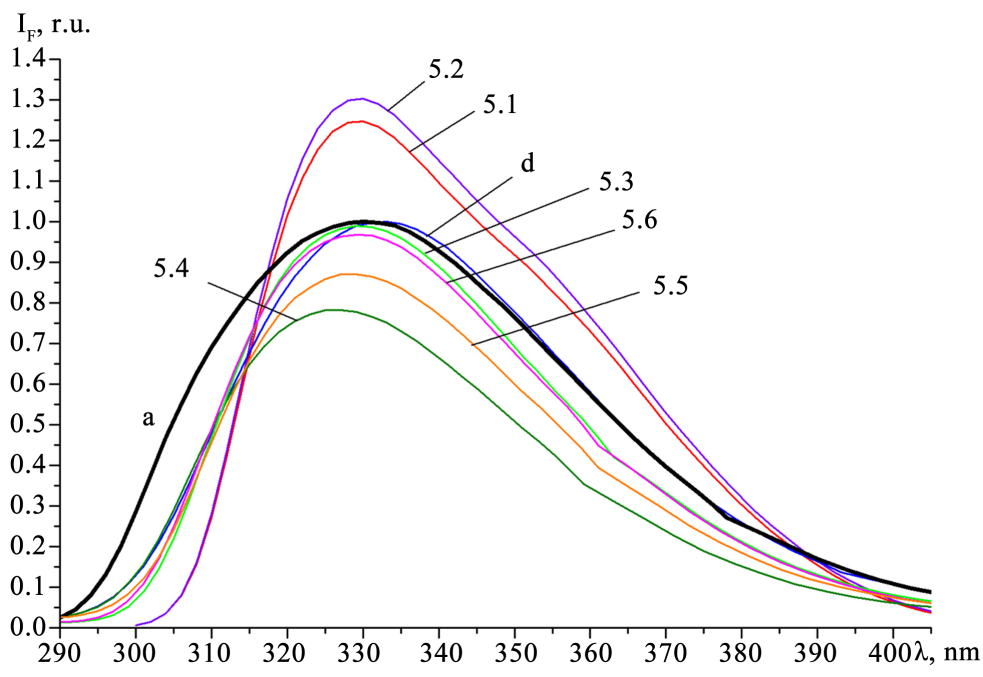

Figure 5. FS of BS of the patient 5 with burn injury, who was hospitalized at Lviv's Communal Clinical Hospital No. 8 in 2017 in dynamics during treatment (5.1-9.02, $5.2-14.02,5.3-22.02,5.4-03.03,5.5-10.03,5.6-31.03$ ), donor (d) and $20 \%$ albumin solution (a), $\lambda_{\mathrm{ex}}=280 \mathrm{~nm}$.

Table 4. Spectral-fluorescence parameters (fluorescence intensity $\left(I_{F}\right)$ and the position of the maximum fluorescence band $\left.\left(\lambda_{\max }\right)\right)$ of blood serum of patient 4 .

\begin{tabular}{ccccccccccc}
\hline $\mathrm{N}$ & $\mathrm{a}$ & 4.1 & 4.2 & 4.3 & 4.4 & 4.5 & $1^{\prime}$ & $2^{\prime}$ & $3^{\prime}$ & $4^{\prime}$ \\
\hline Date & 3.07 & 17.07 & 20.07 & 24.07 & 28.07 & 31.07 & 03.06 & 05.06 & 06.06 & 07.06 \\
$\lambda_{\max } \mathrm{nm}$ & 327 & 335.1 & 335.1 & 333.1 & 333.1 & 335.1 & 335.2 & 335.2 & 334.1 & 331.6 \\
I, r.u. & 1 & 0.98 & 0.83 & 0.76 & 0.78 & 0.96 & 0.63 & 0.43 & 0.14 & 0.40 \\
\hline
\end{tabular}




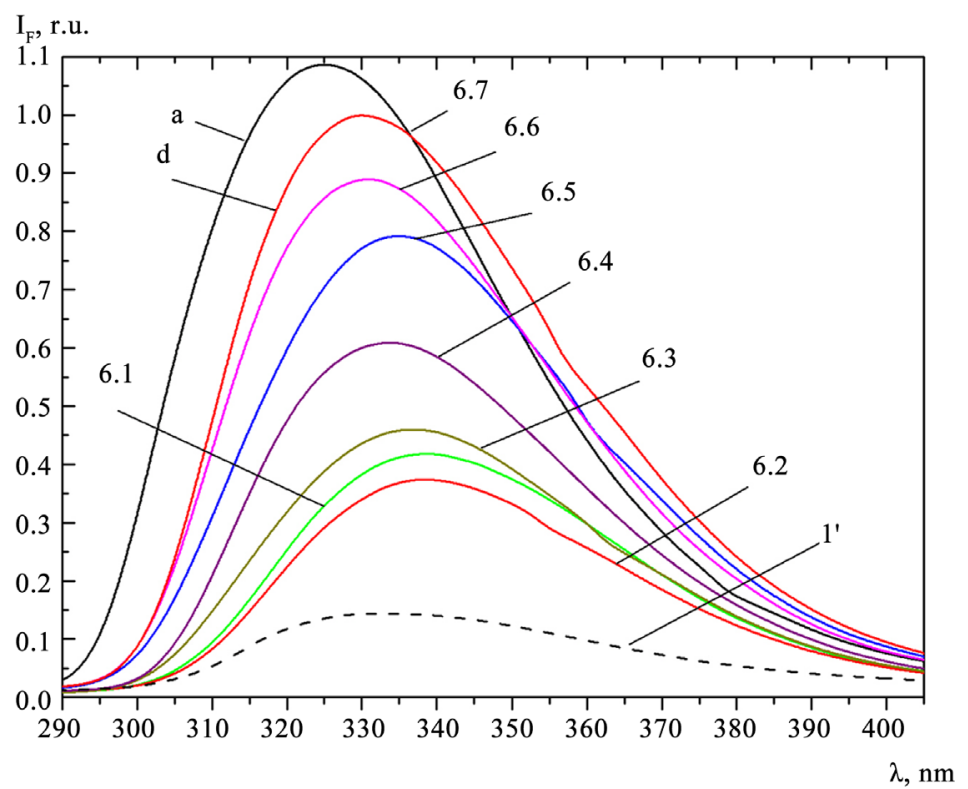

Figure 6. FS of BS of the patient 6 with burn injury, who was hospitalized at Lviv's Communal Clinical Hospital No 8 in 2017 in dynamics during treatment (6.1-9.02, 6.2-14.02, 6.3-22.02, 6.4-27.02, 6.5-03.03, 6.6-10.03, 6.7-31.03), and a patient with sepsis, who was treated in 2002 in Ambulance hospital (1'-03.06) and 20\% albumin solution (a), $\lambda_{\mathrm{ex}}=280 \mathrm{~nm}$.

Table 5. Spectral-fluorescence parameters (fluorescence intensity $\left(I_{F}\right)$ and the position of the maximum fluorescence band $\left(\lambda_{\max }\right)$ ) of blood serum of patient 5 .

\begin{tabular}{ccccccccc}
\hline $\mathrm{N}$ & $\mathrm{a}$ & $\mathrm{d}$ & 5.1 & 5.2 & 5.3 & 5.4 & 5.5 & 5.6 \\
\hline Date & 3.07 & 9.02 & 9.02 & 14.02 & 22.02 & 03.03 & 10.03 & 31.03 \\
$\lambda_{\max } \mathrm{nm}$ & 330.1 & 333.1 & 330 & 330 & 329.1 & 327.1 & 329.1 & 329.1 \\
I, r.u. & 1 & 1 & 1.25 & 1.30 & 0.99 & 0.78 & 0.87 & 0.97 \\
\hline
\end{tabular}

Table 6. Spectral-fluorescence parameters (fluorescence intensity $\left(I_{F}\right)$ and the position of the maximum fluorescence band $\left(\lambda_{\max }\right)$ ) of blood serum of patient 6 .

\begin{tabular}{ccccccccccc}
\hline $\mathrm{N}$ & $\mathrm{a}$ & $\mathrm{d}$ & $1^{\prime}$ & 6.1 & 6.2 & 6.3 & 6.4 & 6.5 & 6.6 & 6.7 \\
\hline Date & 06.06 & 06.06 & 06.06 & 9.02 & 14.02 & 22.02 & 27.02 & 03.03 & 10.03 & 31.03 \\
$\lambda_{\max } \mathrm{nm}$ & 330.1 & 333.1 & 333 & 335.1 & 339.1 & 337 & 334 & 335.1 & 331.1 & 332.0 \\
I, r.u. & 1 & 1 & 0.16 & 0.41 & 0.37 & 0.46 & 0.61 & 0.79 & 0.89 & 0.95 \\
\hline
\end{tabular}

hospitalized in Lviv's Communal Clinical Hospital No 8 in 2017. For the patient with burn area $32 \%$ there was a significant volume of infusion therapy (more than 2 liters daily), so the fluorescence intensity was higher than 1 (curves 5.1 and 5.2). This is consistent with the results of the in vitro study [11]. As the volume of infusion therapy decreased, the fluorescence intensity began to decrease (curve 5.3), which was caused by the leveling off of the effect of concentration quenching. But this decrease was not very noticeable due to the infusion of $10 \%$ albumin solution ( $200 \mathrm{ml}$ once and $100 \mathrm{ml}$ four times), which made it possible to 
replenish the complete albumin in the patient's body, capable of performing its functions [12].

After the cancellation of the infusion of $10 \%$ albumin solution, the fluorescence intensity continued to decrease to $\mathrm{I}_{\mathrm{F}}=0.78$ r.u. (curve 5.4), despite continued antibiotic therapy and antibiotic replacement. Against the background of continued treatment, the following improvement of the patient's condition was noted (curves 5.5 and 5.6), and the fluorescence intensity after completion of the treatment at the time of discharge from the hospital was $\mathrm{I}_{\mathrm{F}}=0.97$ r.u.

FS of BS of the next patient is depicted on Figure 6. The area of the burn surface of this patient was $28 \%$. The volume of infusion therapy of this patient did not differ significantly from the corresponding volume of the previous patient, but the fluorescence intensity of his BS was low (curve $6.1 \mathrm{I}_{\mathrm{F}}=0.41$ r.u., curve 6.2 $I_{F}=0.37$ r.u.). His condition was much more severe, than the condition of the previous patient. During further treatment, including effective antibiotic therapy, as well as infusion of $10 \%$ albumin solution $\left(6^{\text {th }}, 10^{\text {th }}, 15^{\text {th }}, 18^{\text {th }}\right.$ of July, 2017), the fluorescence intensity began to increase from 0.46 r.u. (curve 6.3) up to 0.95 r.u. (curve 6.7). After that, the patient was discharged from the hospital in satisfactory condition.

Thus, we successfully used MFS for patients of the main group to diagnose endogenous intoxication and monitor their condition. The behavior of spectral-fluorescent characteristics of the BS of patients of different severity was under study. Standard treatment regimens were improved. It is noteworthy, that infusions of albumin solution were provided in case the patients felt worse. This ensured a successful treatment process and proper control. We took into account the treatment process under the control of MFS and formed a comparison group of 10 patients, who were treated without supervision within the MFS. But in case of the negative clinical dynamics of the patient's condition, we adjusted the treatment process by including infusions of albumin solution. In all cases, a positive effect was observed due to the changes of treatment tactics. All the patients, $60 \%$ of whom were in serious condition, were discharged from the hospital in satisfactory condition after the successful completion of their treatment.

The comparison group consisted of patients with burn injuries of first-and second-degree burns (type A and B), who was hospitalized at the stage of burn shock in the burn department of Lviv's Communal Clinical Hospital No 8 in 2019-2020. The area of burnt surfaces of patients in the comparison group ranged from $10 \%$ to $35 \% .60 \%$ of the patients in the comparison group were admitted to the hospital in serious condition, and $40 \%$ of the patients were in moderate condition. Now we can focus on a few clinical cases. A 38-year-old patient's occupational injury was treated in the hospital from the $30^{\text {th }}$ of August to the $13^{\text {th }}$ of November, 2019. At the time of admission, the patient's condition was serious. The main diagnosis was second-degree (type A and B) flame burn of $35 \%$ of the head, neck, back and both upper limbs, second-degree burn shock. The patient had a fever and endogenous intoxication. The general blood test revealed leukocytosis with the increased number of rod granulocytes and the in- 
creased rate of erythrocyte sedimentation. The patient underwent a successful surgical treatment. He received an anti-inflammatory, antibacterial, anticoagulant, antifungal and hormonal therapy, infusions of albumin solution (total amount of albumin solution is $700 \mathrm{ml}$ ). The patient also received erythromass $(4$ times) and native plasma (5 times). The daily infusion volume was more than $3000 \mathrm{ml}$. Considering the successful experience of using infusions albumin solution for treating patients with burns in the main group, we applied this experience to the comparison group. The patient's condition was under reliable monitoring. Infusions of albumin solution were provided in the most critical periods of the patient's condition. They made it possible to balance the amount of complete albumin in the BS and improve the patient's condition. After successful completion of the treatment process ( 75 bed days), the patient was discharged from the hospital in satisfactory condition.

Also notable is the clinical case of another patient, a 46-year-old man, who stayed in the hospital from the $19^{\text {th }}$ of October till the $19^{\text {th }}$ of December, 2019 with a $30 \%$-surface burn injury. The patient's diagnosis was hot steam burn second-degree burns (type A and B) of up to $30 \%$ of the torso and both upper limbs. The patient also suffered from a first-degree burn shock.

The patient was hospitalized with acute intense throbbing pain in the affected areas, chills. There was a severing clinical picture of the disease. The patient had endogenous intoxication. He received an appropriate treatment, including surgery (autodermoplasty). He also received anti-inflammatory, antibacterial, infusion therapy with saline and non-saline solutions as well as $20 \%$ albumin solution. The treatment process was accompanied by the positive dynamics of changes in the patient's condition. After 61 bed days, he was discharged from the hospital in satisfactory condition.

Noteworthy is the clinical case of the burn injury of a 51-year-old man, who was hospitalized on the $1^{\text {th }}$ of January, 2020 in serious condition. The diagnosis during hospitalization was second-degree (type A and B) flame burn of $25 \%$ of the head, chest, back, both forearms and hands, and first- and second-degree burn shock. The injury was received at home due to the explosion of a blowtorch. The patient underwent 4 surgeries (autodermoplasties and appendectomy). He also received massive infusion therapy with colloidal, saline and non-saline solutions. He received erythromass 5 times $(213-319 \mathrm{ml})$ and blood plasma 4 times $(180-260 \mathrm{ml})$. The patient also received infusions of $20 \%$ albumin solution $100 \mathrm{ml}$ twice a day 5 times (total amount is $1000 \mathrm{ml}$ ). This contributed greatly to the improvement of his condition. Upon the successful completion of the treatment 76 days later, the patient was discharged home in satisfactory condition to the supervision of a local surgeon.

All patients were observed by us during the entire period of inpatient treatment. After discharge from the hospital, they were under the supervision of a local surgeon.

So, in our research, we proved the successful experience of using MFS to diagnose, control and improve the treatment process for patients with burn inju- 
ries. The experience and skills, gained by using this method, have contributed greatly to the improvement of treatment tactics for severe patients with burn injuries, whose treatment was carried out without the use of MFS. At the same time, further thorough research is very important to improve the diagnosis and treatment tactics, especially during severe purulent-inflammatory diseases, like sepsis.

\section{Conclusions}

Using the method of fluorescence spectroscopy, at the first time, spectral-fluorescence characteristics of blood serum were obtained for the patients with burn injury. They have been found to be the effective markers of the severity of this disease. Their research in dynamics allows to monitor their behavior and to manage effectively the process of treatment. This makes it possible to assess quickly and qualitatively the threat of critical purulent-septic complications in order to adjust treatment and to prevent the development of septic conditions in patients.

It has been justified pathogenetically the positive effect of infusion therapy with albumin solution in the treatment of burn injury. Probable scenarios of behavior of spectral-fluorescence characteristics of BS of patients with burn trauma, depending on the severity of the disease, have been established. Patients with burn injury in serious condition can be used as the model objects for the study of sepsis, including the improvement of the therapeutic tactics of this disease.

\section{Conflicts of Interest}

The authors declare no conflicts of interest regarding the publication of this paper.

\section{References}

[1] Savchyn, V.S. (2014) Features of Reparative Processes in Patients with Deep Burns of the Head and Neck. Archive of Clinical and Experimental Medicine, 23, 149-152.

[2] Savchyn, V.S. (2014) Features of the Inflammatory Response in Burn Head and Neck Injury. Archive of Clinical and Experimental Medicine, 2, 112-113.

[3] Kovalenko, O.M. (2012) Pathogenetic Substantiation of Programs of Surgical Treatment of Children with Common Burns and Their Influence on the Course of Wound Process. Doctor's Thesis, Kyiv, Ukraine, p. 298.

[4] Grizunov, Y.A. and Dobretsov, G.E. (1998) Serum Albumin in Clinical Medicine. Geotar, Moscow, p. 440.

[5] Andreeva, O.L. (2003) Change in the Binding Centers of Serum Albumin in Assessing the State of the Body in Various Pathologies. Doctor's Thesis, Moscow.

[6] Ostapiuk, L. (2019) Diagnostic and Therapeutic Model of Sepsis and Purulent-Inflammatory Diseases. International Journal of Clinical Medicine, 10, 577-595. https://doi.org/10.4236/ijcm.2019.1011047

[7] Herych, I.D., Bulavenko, O.V., Ostapiuk, L.R., Voloshinovskii, A.S. and Myagkota, 
S.V. (2013) Method for Early Diagnosis of Septic Complications by the Method of Fluorescence Spectroscopy. Applicant and Patentee: National Pirogov Memorial Medical University.

[8] Bulavenko, O.V., Ostapiuk, L.R., Rud, V.O., Voloshinovskii, A.S. and Malui, T.S. (2019) Method of Early Diagnosis of Postpartum Purulent-Septic Complications Using the Method of Fluorescence Spectroscopy. Applicant and Patentee: National Pirogov Memorial Medical University.

[9] Savchyn, V.S., Ostapiuk, L.R., Voloshynovskyi, A.S. and Malyi, T.S. (2016) Approbation of the Fluorescence Spectroscopy Method for the Diagnosis of Endogenous Intoxication with Burn Injury. Clinical Surgery, 6, 68-70.

[10] Savchyn, V.S., Ostapiuk, L.R., Voloshinovskii, A.S. and Malyi, T.S. (2019) A New Look at the Diagnosis of Endogenous Intoxication in Patients with Burn Injury. Journal of Hospital Surgery, 1, 20-24.

[11] Bulavenko, O.V., Herych, I.D., Ostapiuk, L.R., Voloshinovskii, A.S., Myagkota, S.V. and Vashchuk, V.V. (2013) Modelling Changes in Blood Serum at Different Diseases and Therapeutic Measures. Biomedical and Biosocial Anthropology, 20, 8-14.

[12] Cherniy V.I. (2017) The Role and Place of Albumin in Modern Infusion Transfusion Therapy. Emergency Medicine, 1, 1-11.

https://doi.org/10.22141/2224-0586.1.80.2017.94448 